\title{
ENDOGENOUS PRODUCTIVITY AND EQUALITY OF OPPORTUNITY
}

\author{
EFthymios Athanasiou \\ efthymios.athanasiou@uclouvain.be
}

\begin{abstract}
We consider a production economy 'à la Mirrlees' to be one in which the earning capability of individuals is endogenous. Individuals are heterogeneous with respect to their preferences and their propensity to benefit from a given investment in human capital. We look for allocation rules satisfying properties that capture the objective of equalizing opportunities. We characterize four allocation rules that both encompass different perspectives of equality of opportunity and justify different levels of public intervention.
\end{abstract}

\section{INTRODUCTION}

Equality of opportunity does not a priori preclude the prevalence of an unequal distribution of social outcomes. Roemer[25] invokes the 'non-discrimination' principle which asserts that only relevant attributes should matter in competing for a position in the social hierarchy. Social outcomes should not be determined by supposedly irrelevant characteristics, such as race, sex and heritage. Yet pinning down the characteristics that 'should' matter is far from staightforward. Indeed, in order to define Equality of Opportunity, one needs to understand the nature of individual differences.

The model we put forward is nothing more than an extension of Mirrlees[15]. In contrast to Mirrlees, individuals differ in their preferences and productivities are endogenous. We incorporate into the model an earnings function that transforms investment in human capital into earnings capabilities. Individuals are characterized by different innate abilities and thus have different earnings possibilities. However, a priori, these initial discrepancies that may reflect family background and genetic predisposition, among other things, need not translate into an unequal distribution of marginal productivities. The actual productivity with which an agent may transform labour into the consumption good will be determined, inter alia, by the amount he chooses to 'invest in himself'.

At first glance, this presents the policy maker with a range of new possibilities. For instance, one may opt for equalizing productivities or strive to guarantee everyone a certain amount of investment in human capital. Although, throughout the paper, we insist on a first best approach, we find that such solutions are excluded if one is to respect Pareto Efficiency. In Figure 1, we introduce a rough version of the model. On the left hand side we have the standard Mirrlees consumption space, comprising labour and a consumption good, such as money. On the right hand side we plot two earnings functions, reflecting a supposition that individual $\mathrm{A}$ is more talented than individual $\mathrm{B}$. If we were to impose a human capital investment profile $\left(m_{A}, m_{B}\right)$ and then bill each individual for the expenditure $m_{A}+m_{B}$ equally, we would have $\mathrm{A}$ and $\mathrm{B}$ facing the same budget line (assuming a constant marginal productivity technology). We have equalized consumption opportunities. Suppose, then, that we let individuals maximize their preferences. The resulting allocation $\left(z_{A}, z_{B}\right)$ is not Pareto Efficient: The allocation $\left(z_{A}^{\prime}, z_{B}\right)$ Pareto dominates $\left(z_{A}, z_{B}\right)^{1}$.

\footnotetext{
${ }^{1}$ To obtain this allocation we invest $\left(0, m_{B}^{\prime}\right)$ and charge individuals A and B zero and $m_{B}^{\prime}=m_{A}+m_{B}$ respectively. Individual A's budget line is in effect the labour axis.
} 


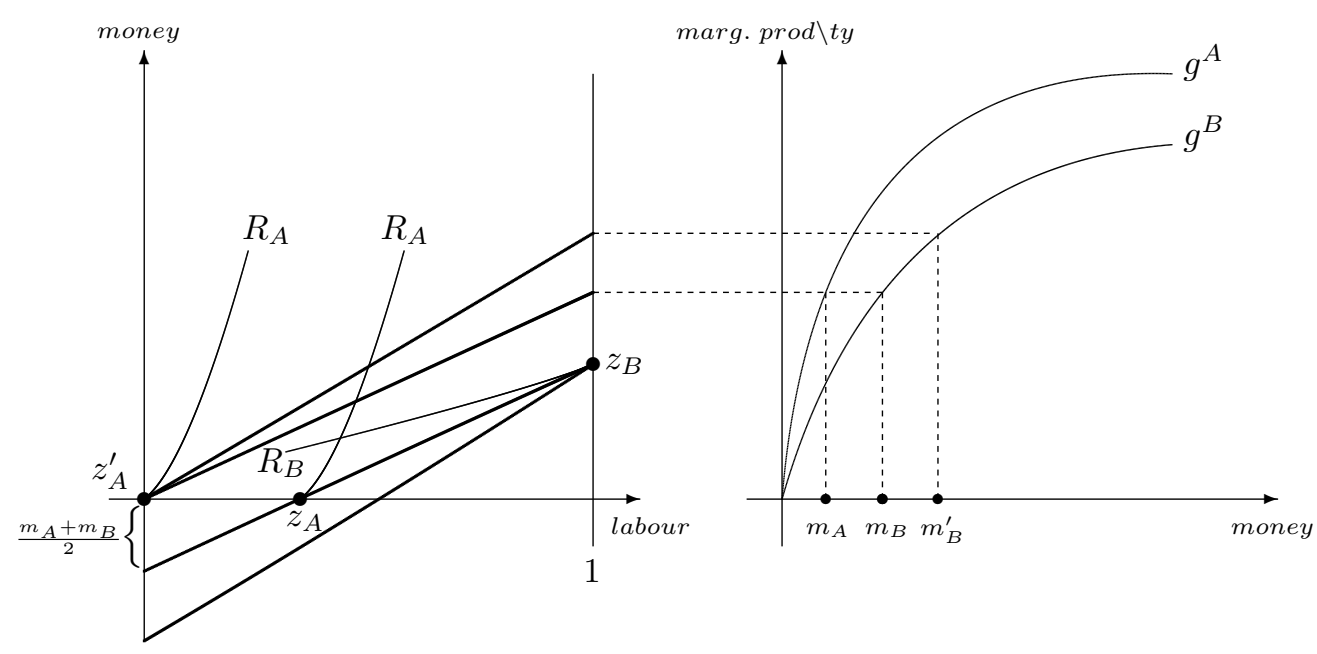

Figure 1. An investment profile that equalizes opportunities.

Therefore, we have to abandon the most intuitive interpretation of equality of opportunity, that of 'leveling the playing field', and examine more subtle factors. It is insights such as this that make the first-best analysis an exercise worth a paper of its own. It is more than a prerequisite for introducing second-best considerations. It serves to clarify and fix ideas regarding equality of opportunity.

Our departing premise is that two broad categories of individual differences, preferences and innate abilities, warrant differential treatment in the context of equality of opportunity. This can be best illustrated by an example. Suppose Sarah and Matt are equally hardworking lawyers. However, Sarah has secured a better job than Matt, because her mother, a well established lawyer, intervened on her behalf. Sarah enjoys a higher income than Matt thanks to her family background. Matt is not to blame for falling short of Sarah. He is not accountable for the fact that his parents are, say, doctors and therefore could not help him in the job market. The principle of compensation dictates that the difference in welfare between Sarah and Matt should be eliminated. Let us now consider a different story, that of Maria and Nelson. Both are alike in all respects except that Nelson is not as hard a worker as Maria is. Naturally Maria earns more than Nelson. Both Maria and Nelson are responsible for their disposition towards labour. In recognition of this fact, the principle of responsibility dictates that the difference in income between Maria and Nelson be preserved ${ }^{2}$. In brief, the differences in social outcomes that we should strive to correct are those solely due to differences in innate abilities. On the contrary, there is no reason to amend differences in social outcomes that are solely the result of differences in preferences. The two objectives, Compensation and Responsibility, will play a crucial role in the definition of Equality of Opportunity. This distinction, following Fleurbaey[6], can be viewed as a particular manifestation of Roemer's non-discrimination principle.

A series of papers (see Fleurbaey and Maniquet[10] for a review), in different contexts, demonstrate that Compensation, Responsibility and Pareto Efficiency clash with one another. One cannot fully fulfill all these separate requirements. In the framework of our

\footnotetext{
${ }^{2}$ See Arneson[2] for a discussion on whether individual preferences develop under circumstances that are under the control of individuals. In this present work, assuming responsibility should be interpreted to mean that one cannot establish any claim over resources on the basis of one's preferences.
} 
model we reiterate this conclusion, perhaps more emphatically, as we find that the incompatibility holds, independently of the earnings function. What ought to be an appropriate compromise is far from apparent. One of the two principles needs to be abandoned in order to accommodate the other. Any point of view regarding this trade-off is treated as a legitimate view of Equality of Opportunity. Thus, Equality of Opportunity is defined as a moral stance in the face of an ethical difficulty.

Fleurbaey and Maniquet [7],[8] are the two contributions which come closest to our approach. Their model involves a production economy with exogenous productivities. They are the first to recognize the incompatibility between Compensation and Responsibility in the context of a production economy. Roemer[25] attempts a similar exercise in which he employs conceptual tools that appear to derive from the ideas of Compensation and Responsibility. He adopts, however, a different model. The aim of this paper is to revisit the conclusions of these works, drawing new insights and recasting certain solutions built on new foundations.

The paper proceeds as follows. Section 1 presents the model while section 2 elaborates on the consequences of Pareto efficiency. Section 3 presents the axioms that encapsulate the main ideas we have addressed in this brief introduction. Section 4 proposes solutions and discusses their implications. Section 5 sums up and discusses the potential extension to second best. In the appendix we prove propositions 3 and 4 .

\section{THE MODEL}

Let $\mathbb{N}_{+}$be the set of positive integers and $2^{\mathbb{N}_{+}}$denote the set of all finite and non-empty subsets of $\mathbb{N}_{+}$. A population of agents is some $N \in 2^{\mathbb{N}_{+}}$. Let $\mathcal{R}$ denote the set of complete, transitive, convex, monotonic ${ }^{3}$ and continuous preference relations over bundles $z \equiv(l, c) \in$ $Z \equiv[0,1] \times \mathbb{R}_{+}$, where $l$ stands for labour time, $c$ denotes consumption and $\mathbb{R}_{+}$is the nonnegative real line. Individuals are endowed with a preference $R_{i} \in \mathcal{R}$. The strict preference and indifference counterparts of $R_{i}$ are $P_{i}$ and $I_{i}$ respectively.

For all $N \in 2^{\mathbb{N}_{+}}$and all $i \in N$, let $a_{i} \in \mathbb{R}_{+}$determine the individual's innate ability, or, talent. If for some $i, j \in N$ we have that $a_{i}>a_{j}$, we infer that individual $i$ is more talented than individual $j$. Each individual $i \in N$ may forgo consumption to invest an amount $k_{i} \in$ $\mathbb{R}_{+}$in order to build up his productivity. The earnings function is captured by $g: \mathbb{R}_{+}^{2} \rightarrow \mathbb{R}_{+}$ that transforms pairs of $\left(k_{i}, a_{i}\right)$ into a constant individual marginal productivity. We require that the earnings function satisfy the following properties. Assumption 1, as general as it may be, allows for the possibility of equalizing marginal productivities.

Assumption 1. The funcion g satisfies the following properties for all $a>0$ :

(a) it is twice continuously differentiable in $k$,

(b) for all $k \geq 0$

$$
\frac{\partial g(k, a)}{\partial k}>0, \quad \frac{\partial^{2} g(k, a)}{\partial k^{2}}<0, \quad g(0, a)=0,
$$

(c) $a>a^{\prime}$ implies $g(k, a)>g\left(k, a^{\prime}\right)$, for all $k>0$,

There is mild empirical evidence in support of Assumption 1 (Hanushek[11], Angrist and Krueger[1]). Loury[12] and Becker et al.[3] develop theoretical models that involve earnings functions similar to the one we propose.

Let $\mathcal{G}$ be the family of functions satisfying Assumption 1. For all $N \in 2^{\mathbb{N}_{+}}$define $R_{N} \equiv$ $\left(R_{i},\right)_{i \in N}, a_{N} \equiv\left(a_{i}\right)_{i \in N}$. An economy $e_{N}$ is described by the following profile:

$$
e_{N}=\left(R_{N}, a_{N}, g\right) \in \mathcal{E} \equiv \bigcup_{N \in 2^{\mathbb{N}_{+}}}\left(\mathcal{R}^{|N|} \times \mathbb{R}_{+}^{|N|} \times \mathcal{G}\right) .
$$

\footnotetext{
${ }^{3}$ With respect to leisure and consumption.
} 
Slightly abusing the notation, we will sometimes treat $R_{N}, a_{N}$ as sets of elements, writing for instance $a_{i} \in a_{N}$ and $\min a_{N} \equiv\left\{a_{i} \in a_{N}: a_{i} \leq a_{j}\right.$, for all $\left.a_{j} \in a_{N}\right\}$.

Agents are endowed with one unit of leisure and zero units of the consumption good. Any amount of investment in human capital will be paid out of labour income. The consumption and investment goods are perfect substitutes. We impose no physical constraint on the amount that can be invested. Imagine perfect credit markets that lend amounts of the investment good at no interest. Returns to scale are constant ${ }^{4}$. Therefore, the feasibility constraint for any economy is given by:

$$
\sum_{i \in N}\left(c_{i}+k_{i}\right) \leq \sum_{i \in N} g\left(k_{i}, a_{i}\right) l_{i}
$$

An allocation $z_{N} \equiv\left(l_{i}, c_{i}\right)_{i \in N} \in Z^{|N|}$ is feasible for $a_{N}, g$ if it satisfies the feasibility constraint. The set of feasible allocations for a given economy $e_{N} \in \mathcal{E}$ is denoted $Z(e)$. An allocation rule $\varphi$ is a correspondence that assigns a non-empy subset of $Z(e)$ to each economy in the domain $\mathcal{E}$.

\section{Efficient Educational Outcomes}

The purpose of this section is to investigate the implications of Pareto Efficiency. We require that all the allocation rules we consider henceforth satisfy the following axiom.

Axiom 1. An allocation rule $\varphi$ satisfies Pareto Efficiency $(\mathrm{PE})$ if and only if for all $e_{N} \in \mathcal{E}$, all $z_{N} \in \varphi\left(e_{N}\right)$ and all $z_{N}^{\prime} \in Z\left(e_{N}\right)$,

$$
z_{i}^{\prime} R_{i} z_{i}, \forall i \in N \Rightarrow z_{i}^{\prime} I_{i} z_{i}, \forall i \in N
$$

A utility representation of some $R_{i} \in \mathcal{R}$ is denoted $u_{i}(c, l)$. The necessary (and under our assumptions on preferences and technology, sufficient) conditions for Pareto Efficiency ${ }^{5}$ in the interior of the consumption set are:

$$
\begin{gathered}
-\frac{\frac{\partial u_{i}}{\partial l_{i}}}{\frac{\partial u_{i}}{\partial c_{i}}}=g\left(k_{i}, a_{i}\right) \quad \forall i \in N, \\
\frac{\partial g\left(k_{i}, a_{i}\right)}{\partial k_{i}}=\frac{1}{l_{i}} \quad \forall i \in N .
\end{gathered}
$$

Conditions (1) and (2) relate to the following lemma.

Lemma 1. Every Pareto Efficient allocation can be decentralized through a vector of lump sum transfers.

Proof. An allocation $z_{N}^{*}$ and a vector $k_{N}^{*}$ constitute an equilibrium in the economy $e_{N} \in \mathcal{E}$ if for all $i \in N$

\footnotetext{
${ }^{4}$ The story we are unraveling is consistent with an OLG model in which individuals live two periods, investment in human capital occurs when young, consumption when old, and the distribution of innate abilities remains constant across different generations.

${ }^{5}$ To determine the set of allocations that satisfy Pareto Efficiency we solve the following problem:

$$
\max _{\left\{c_{i}, l_{i}, k_{i}\right\}_{i \in N}} u_{1}\left(c_{1}, l_{1}\right)
$$
}

subject to

$$
\begin{gathered}
u_{i}\left(c_{i}, l_{i}\right) \geq \overline{u_{i}} \quad \forall i \in N \backslash\{1\} \\
\sum_{i \in N}\left[g\left(k_{i}, a_{i}\right) l_{i}\right] \geq \sum_{i \in N}\left(c_{i}+k_{i}\right) \\
c_{i}, k_{i} \geq 0 \text { and } 0 \geq l_{i} \geq 1 \quad \forall i \in N
\end{gathered}
$$


I $k_{i}^{*} \in \operatorname{argmax}\left\{g\left(k_{i}, a_{i}\right) l_{i}^{*}-k_{i}\right\}$ and

II $z_{i}^{*}$ maximizes $R_{i}$ in

$$
\left\{\left(l_{i}, c_{i}\right) \in[0,1] \times \mathbb{R}_{+}: c_{i} \leq g\left(k_{i}^{*}, a_{i}\right) l_{i}-k_{i}^{*}\right\} .
$$

By Assumption 1, the function $g$ is strictly concave and the production technology is linear in $l_{i}$. Therefore, the F.O.C.s associated with I,II are sufficient for optimality. Finally, the F.O.C.s of I,II coincide with the F.O.C.s of the Pareto Efficiency problem. Therefore, if an allocation $z_{N}$ is Pareto Efficient then $z_{N}$ along with the associated vector $k_{N}$ constitutes a solution to I,II.

Lemma 1 tells us that if an allocation rule $\varphi$ satisfies Pareto Efficiency, then for any $e_{N} \in$ $\mathcal{E}$ and for any $z_{N} \in \varphi\left(e_{N}\right)$ there exists a competitive equilibrium that decentralizes $z_{N}$. Consequently, for all admissible economies, there exists a one-to-one correspondence between the set of competitive equilibria and the set of Pareto Efficient allocations.

It is of interest to look into condition (2) in more detail. It is particular to the model. Its Pareto Efficiency interpretation is that consumption possibilities should not be wasted. From an equilibrium perspective it accomplishes the maximization of earnings (measured in units of the consumption good). To take the intuitive approach, suppose that the individual faces the problem of maximizing his earnings, after bearing the cost of his education, constrained to work an $l^{*}$ amount of time:

$$
\max _{k \geq 0} g(k, a) l-k \quad \text { subject to } \quad l=l^{*} .
$$

The value function that corresponds to this problem is

$$
v\left(l^{*} ; s\right) \equiv g\left(k\left(l^{*}\right), a\right) l^{*}-k\left(l^{*}\right),
$$

where $k\left(l^{*}\right)$ solves the equation $\frac{d g(k, a)}{d k} l^{*}-1=0$, i.e. equation (2). We call this value function the outcome frontier. It is the locus of $(l, c)$ bundles that lie on the frontier of the consumption possibilities of the individual.

Lemma 2. The outcome frontier is increasing and convex.

Proof. $\quad$ From the envelope theorem

$$
\frac{\partial}{\partial l^{*}} v\left(l^{*}, a\right)=g\left(k\left(l^{*}\right), a\right) .
$$

By Assumption 1, clause (b), the proof is complete.

The real bite of Pareto Efficiency stems from the following lemma. In the set of efficient allocations, talent differentials translate bluntly into different outcome frontiers that dominate one another.

Lemma 3. For any pair $i, j \in N$, with $a_{i}>a_{j}$, the outcome frontier of individual $i$ lies strictly above that of individual $j$, except at the origin, where they coincide.

Proof. Let $a>a^{\prime}$. By Assumption 1, clause (c), $g(k, a)>g\left(k, a^{\prime}\right)$, for all $k>0$. Therefore, $g(k, a) l-k>g\left(k, a^{\prime}\right) l-k$, for all $l, k$ positive. Hence, $v(l, a)>v\left(l, a^{\prime}\right)$, for all $l>0$. Finally, if $l=0$, then $v(l, a)=0$, for all $a>0$, since otherwise optimality would be contradicted.

The above lemmata and in particular lemma 3, precisely focus the message we attempted to convey with the example we invoked in the introduction. In any generic economy, 'equalizing opportunities' in the straightforward sense conflicts with Pareto Efficiency: no sensible policy can force the outcome frontiers which individuals face to coincide. It is impossible to achieve 

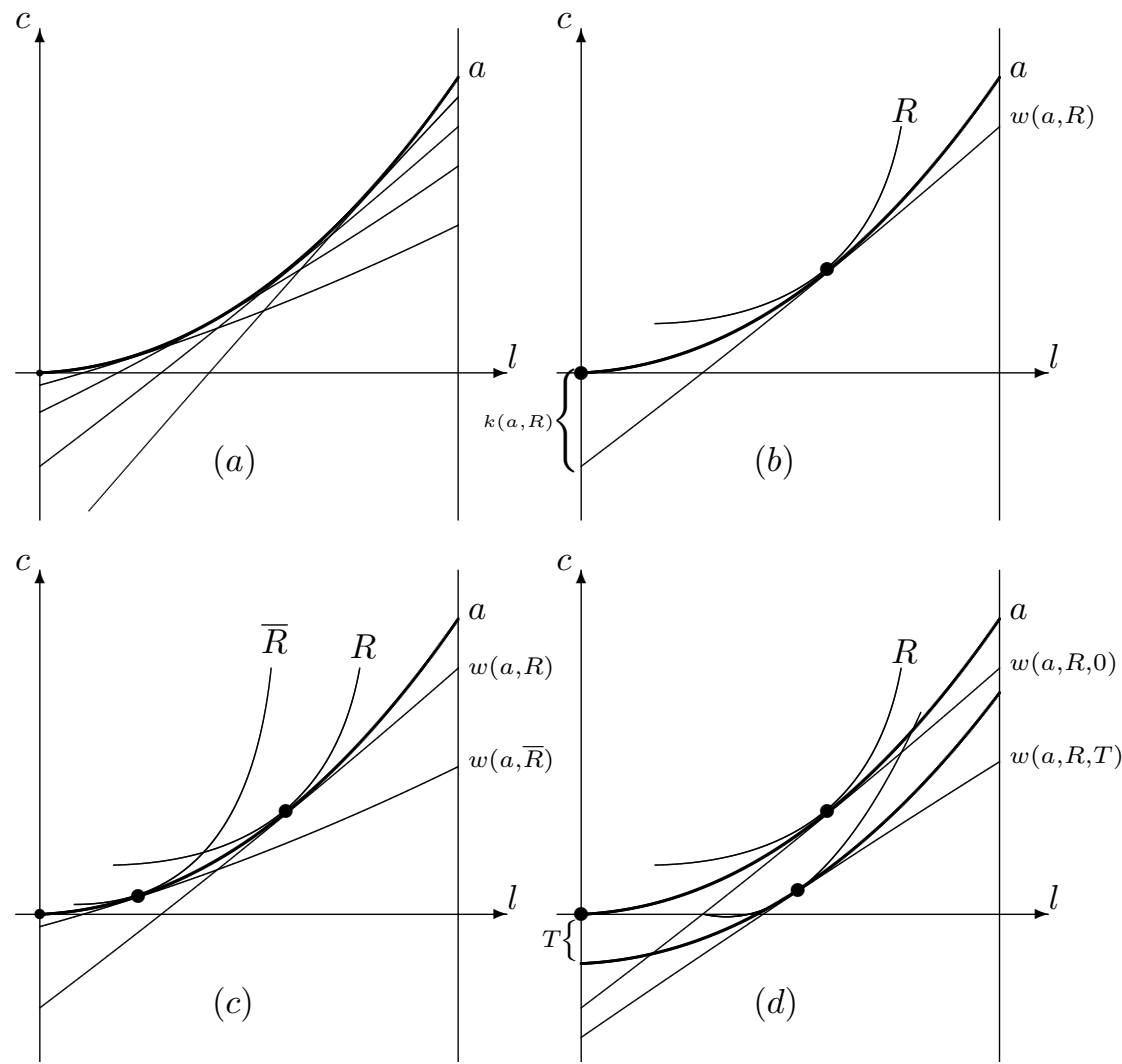

Figure 2. Pareto Efficiency and lump-sum transfers.

an efficient and fair outcome simply by organizing the investment profile $k_{N}$ in a way that disregards information on preferences and innate abilities. However, Pareto Efficiency is compatible with lump-sum transfers, as lemma 1 stresses. Such transfers can be seen as a means of redistributing opportunities or consumption possibilities among agents. In fact, as we shall see shortly, underlying any Pareto Efficient allocation rule, is a vision on how these transfers should be carried out and the purpose they should strive to fulfill.

In figure 2 we illustrate these observations. Part (a) of the figure illustrates the fact that the outcome frontier can be viewed as a menu of marginal productivities, or equivalently, budget lines. The tangent at each point of the outcome frontier has a slope equal to the marginal productivity. Alternatively, these are the wages or prices that decentralize an efficient allocation. The bigger a propensity for effort an individual has, the higher the wage that he will be paid for her labour. Part (b) of the figure reminds us that an efficient bundle lies on the outcome frontier and, moreover, at a point where the indifference curve of the individual is supported by it. Hence marginal productivity depends on both innate ability and preferences. In addition, the distance between the origin and the intercept of the tangent with the horizontal axis is the amount $k$ invested in human capital. Part (c) of the figure depicts the efficient bundles associated with the case of two individuals with the same innate ability, yet different preferences. This particular allocation involves zero transfers.

Graphically ${ }^{6}$, lump-sum transfers amount to a vertical translation of the outcome frontier as depicted in part (d) of figure 2. Note that a lump-sum transfer will affect the actual

\footnotetext{
${ }^{6}$ In all the diagrams, we choose to denote the outcome frontier by the parameter $a$. The proper expression $V(l ; a, T)$ is rather heavy. We hope this does not generate confusion.
} 
wage rate an individual faces (unless her preferences are quasi-linear) and thus another determinant along with preferences and innate ability enters the picture. ${ }^{7}$ Algebraically we could write $v(l ; a)-T$, with $T \in \mathbb{R}$. Let $V(l ; a, T) \equiv v(l ; a)-T$. The Outcome Set, that is, the set of feasible outcomes for an individual with talent $a \in \mathbb{R}_{+}$, facing a lump-sum tax $T \in \mathbb{R}$, is

$$
O S(a, T)=\left\{(l, c) \in[0,1] \times \mathbb{R}_{+}: c \leq V(l ; a, T)\right\} .
$$

Before we move on, let us introduce some further pieces of notation. We denote the maximal elements of the Outcome Set, from the point of view of an individual $i \in N$, with preference relation $R_{i}$, by $m\left(R_{i}, O S(a, T)\right)^{8}$. In addition, let $N(a) \equiv\left\{i \in N: a_{i}=a\right.$ for some $\left.a \in \mathbb{R}_{+}\right\}$.

Definition. For all $e_{N} \in \mathcal{E}$ and all $z_{N} \in Z\left(e_{N}\right)$ define $t\left(z_{N}\right)=\left(t_{i}\left(z_{N}\right)\right)_{i \in N}$ to be such that, for all $i \in N$ :

$$
t_{i}\left(z_{N}\right)=\min \left\{t \in \mathbb{R}: z_{i} \in O S\left(a_{i}, t\right)\right\} .
$$

For all $e_{N} \in \mathcal{E}$ and all $z_{N} \in Z\left(e_{N}\right)$, the set $\min \left\{t \in \mathbb{R}: z_{i} \in O S\left(a_{i}, t\right)\right\}$ is non-empty and contains only one element. Hence, to any feasible allocation we may assign a vector of lump-sum transfers. Of course, this vector decentralizes $z_{N}$ only if $z_{N}$ is Pareto Efficient.

\section{THE AXIOMS}

Axioms 2-4, presented below, proceed from Fleurbaey[6]. We use them to conceptualize Equality of Opportunity, as already noted. We begin with the idea of Compensation for differences in innate ability. We require that no advantage be grounded on parameters that are beyond individual accountability. Individuals with the same preferences should enjoy the same level of satisfaction. Such individuals may potentially differ only with respect to innate ability.

Axiom 2. An allocation rule $\varphi$ satisfies Equal Welfare for Equal Preference (EWEP) if and only if for all $e_{N} \in \mathcal{E}$, all $z_{N} \in \varphi\left(e_{N}\right)$ and all $i, j \in N$

$$
R_{i}=R_{j} \Rightarrow z_{i} I_{i} z_{j} .
$$

The following two axioms capture the idea of Responsibility. Any two individuals with the same innate ability should have the same consumption opportunities or, more practically, should end up on the same outcome frontier. Differences in preferences alone are not a legitimate reason to elicit unequal treatment. From an ethical point of view, individuals should be neither rewarded nor punished for their preferences. In plain terms, axiom 3 requires that individuals with the same ability should face the same lump-sum transfer.

Axiom 3. An allocation rule $\varphi$ satisfies Equal Outcome Frontier for Equal Ability (EFEA) if and only if for all $e_{N} \in \mathcal{E}$, all $z_{N} \in \varphi\left(e_{N}\right)$ and all $i, j \in N$

$$
a_{i}=a_{j} \Rightarrow t_{i}\left(z_{N}\right)=t_{j}\left(z_{N}\right) .
$$

A weaker idea (Fleurbaey and Maniquet[7]) would be to posit no envy among individuals who share the same talent. Indeed, it is not necessary for two equally able individuals to face the same transfer in order for the following axiom to be satisfied.

\footnotetext{
${ }^{7}$ If one wishes to interpret human capital technology as underlying a process of education, a word of caution is in order. The model is amenable to both the private and public schooling interpretations. In the former case the transfer should be seen as tax liability, while in the latter case, as a rebate on educational expenditures.

${ }^{8}$ We will write $z R_{i} m\left(R_{i}, B\right)$ to assert that, in fact, $z R_{i} \tilde{z}, \forall \tilde{z} \in m\left(R_{i}, B\right)$
} 
Axiom 4. An allocation rule $\varphi$ satisfies No Envy among the Equally Able (NEEA) if and only if for all $e_{N} \in \mathcal{E}$, all $z_{N} \in \varphi\left(e_{N}\right)$ and all $i, j \in N$

$$
a_{i}=a_{j} \Rightarrow z_{i} R_{i} z_{j} \text { and } z_{j} R_{j} z_{i} \text {. }
$$

The axioms that follow do not directly relate to Equality of Opportunity. However, Equality of Opportunity as an ethical goal does not justify all possible means. We maintain that an allocation rule should refrain from exploiting individuals. Put simply, we ask that the poorly endowed establish a right over a policy that, while aiming at improving their situation, at the same time does not 'exploit' the talented. Axioms 5 and 6 that follow defend the idea of collective ownership of the potential that the parameter $a_{i}$ associates with each individual. It relates to Rawls[23] who claims that no one 'deserves' his place in the distribution of innate abilities. Thomson[20] and Roemer[24] proposed an axiom that manifests the Ralwsean principle concretely. Ability Solidarity requires that either we all benefit or we all suffer from any arbitrary change in the vector of innate abilities. The consequences of such a change should be born collectively.

Axiom 5. An allocation rule $\varphi$ satisfies Ability Solidarity (AS) if and only if for all $e_{N}=$ $\left(R_{N}, a_{N}, g\right) \in \mathcal{E}$, all $e_{N}^{\prime}=\left(R_{N}, a_{N}^{\prime}, g\right) \in \mathcal{E}$, all $z_{N} \in \varphi\left(e_{N}\right)$ and all $z_{N}^{\prime} \in \varphi\left(e_{N}^{\prime}\right)$

either $z_{i} R_{i} z_{i}^{\prime}$ for all $i \in N$ or $z_{i}^{\prime} R_{i} z_{i}$ for all $i \in N$.

Ability Solidarity can be interpreted as the impetus that drives an allocation rule towards redistribution. We will see that it takes a clear stand in favor of Compensation objectives. However, Ability Monotonicity, defined below (which is implied by Ability Solidarity, together with Pareto Efficiency), is fully compatible with Equal Outcome Frontier for Equal Ability, so much so as to be satisfied by a laissez-faire like allocation rule. It requires that everyone benefit whenever the vector of innate abilities (weakly) increases. It should be interpreted as an axiom that prevents the exploitation of the less talented. ${ }^{9}$

Axiom 6. An allocation rule $\varphi$ satisfies Ability Monotonicity (AM) if and only if for all $e_{N}=\left(R_{N}, a_{N}, g\right) \in \mathcal{E}$, all $e_{N}^{\prime}=\left(R_{N}, a_{N}^{\prime}, g\right) \in \mathcal{E}$, all $z_{N} \in \varphi\left(e_{N}\right)$, all $z_{N}^{\prime} \in \varphi\left(e_{N}^{\prime}\right)$ and all $i \in N$

$$
a_{N}^{\prime} \geq a_{N} \Rightarrow z_{i}^{\prime} R_{i} z_{i} .
$$

While we endorse solidarity, still, we would like to avoid what Dworkin[4][5] calls the 'slavery of the talented'. We would not allow for transfers schemes that would cause the talented to 'resent their gift'. Limited Self-Ownership of Ability requires that a transfer of resources not go as far as making anyone in the society envious of some least able individual's consumption possibilities.

Axiom 7. An allocation rule $\varphi$ satisfies Limited Self-Ownership of Ability (LSOA) if and only if for all $e_{N} \in \mathcal{E}$, all $z_{N} \in \varphi\left(e_{N}\right)$, all $i \in N$ and all $j \in N\left(\min a_{N}\right)$

$$
z_{i} R_{i} m\left(R_{i}, O S\left(a_{j}, t_{j}\left(z_{N}\right)\right)\right)
$$

Minimal Self-Ownership of Ability is a variant of the same idea. No individual should be worse off than he would be if he belonged to the set $N\left(\min a_{N}\right)$ and, moreover, there were no transfers.

Axiom 8. An allocation rule $\varphi$ satisfies Minimal Self-Ownership of Ability (MSOA) if and only if for all $e_{N} \in \mathcal{E}$, all $z_{N} \in \varphi\left(e_{N}\right)$, all $i \in N$ and all $j \in N\left(\min a_{N}\right)$

\footnotetext{
${ }^{9}$ We write $a_{N}^{\prime} \geq a_{N}$ to mean $a_{i}^{\prime} \geq a_{i}$, for all $i \in N$.
} 


$$
z_{i} R_{i} m\left(R_{i}, O S\left(a_{j}, 0\right)\right)
$$

Axioms 7 and 8 encompass mild libertarian objectives. They are probably too weak for the taste of philosophers like Nozick[19], who invoke the Kantian principle that one should not serve as a means for another. They can be interpreted as lower bounds. Moulin and Roemer[18] propose axioms that relate to ours. Since we employ a model with constant returns to scale, we do not use bounds à la Moulin[16],[17]. It should be stressed, finally, that Limited and Minimal Self-Ownership of Ability, are logically unrelated. In particular, having all the least able receive a negative transfer is compatible with the former, yet violates the latter. Both of them, however, are a consequence of 'almost the same' list of axioms, as Propositions 1 and 2 illustrate.

Proposition 1. If an allocation rule $\varphi$ satisfies Pareto Efficiency, Equal Outcome Frontier for Equal Ability and Ability Monotonicity then it satisfies Minimal Self-Ownership of Ability.

Proof. Let $\varphi$ satisfy the axioms. Consider $e_{N}=\left(R_{N}, a_{N}, g\right) \in \mathcal{E}$ and let $e_{N}^{\prime}=$ $\left(R_{N}, s_{N}^{\prime}, g\right) \in \mathcal{E}$ be such that $a_{N}^{\prime}=(\underline{a}, \underline{a}, \ldots, \underline{a})$, where $\underline{a} \equiv \min a_{N}$. Suppose $z_{N} \in \varphi\left(e_{N}\right)$. By PE and EFEA, for any $z_{N}^{\prime} \in \varphi\left(e_{N}^{\prime}\right)$ it must be $z_{i}^{\prime} \in m\left(R_{i}, O S\left(\min a_{N}, 0\right)\right)$, for all $i \in N$. By AM, for all $i \in N, z_{i} R_{i} z_{i}^{\prime}$. Hence, we have recovered MSOA.

To recover Limited Self-Ownership of Ability we need to enrich the list of axioms in Proposition 2. In particular, we will appeal to Replication Invariance, which requires that the prescription of the allocation rule carry through when the economy is replicated. In the formal definition we follow Maniquet and Sprumont[14].

Definition. Let $\rho$ be a positive integer. We will say that an economy $e_{N^{\rho}}=\left(R_{N^{\rho}}, a_{N^{\rho}}, g\right) \in$ $\mathcal{E}$ is a $\rho$-replica of $e_{N}=\left(R_{N}, a_{N}, g\right) \in \mathcal{E}$ if and only if the following conditions hold:

1 The function $g$ is the same in both economies,

2 there exists a mapping $\xi: N^{\rho} \rightarrow N$ such that for all $i \in N,\left|\xi^{-1}(i)\right|=\rho$,

3 for all $i \in N$ and all $j \in \xi^{-1}(i), R_{j}=R_{i}$ and $a_{j}=a_{i}$.

Similarly, we may define the $\rho$-replica of an allocation. For any $e_{N}, e_{N} \rho$ and some $z_{N} \varphi\left(e_{N}\right)$, let $z_{N^{\rho}}^{\rho}$ be such that $z_{i}=z_{j}^{\rho}$, for all $i \in N$ and $j \in \xi^{-1}(j)$.

Axiom 9. An allocation rule $\varphi$ satisfies Replication Invariance (RI) if and only if for all $e_{N}=\left(R_{N}, a_{N}, g\right) \in \mathcal{E}$ and all $e_{N^{\rho}}=\left(R_{N^{\rho}}, a_{N^{\rho}}, g\right) \in \mathcal{E}$ such that $e_{N^{\rho}}$ is a $\rho$-replica of $e_{N}$

$$
z_{N} \in \varphi\left(e_{N}\right) \Rightarrow z_{N^{\rho}}^{\rho} \in \varphi\left(e_{N^{\rho}}\right) \text {. }
$$

Proposition 2. If an allocation rule $\varphi$ satisfies Pareto Efficiency, Equal Outcome Frontier for Equal Ability, Ability Monotonicity and Replication Invariance then it satisfies Limited Self-Ownership of Ability.

Proof. Let $\varphi$ satisfy the axioms. Suppose that there exists $e_{N}=\left(R_{N}, a_{N}, g\right) \in \mathcal{E}$, $z_{N} \in \varphi\left(e_{N}\right), k \in N$ and $j \in N\left(\min a_{N}\right)$ such that

$$
m\left(R_{k}, O S\left(a_{j}, t_{j}\left(z_{N}\right)\right)\right) P_{k} z_{k} .
$$

Define $t^{*} \in \mathbb{R}$ to be such that

$$
m\left(R_{k}, O S\left(a_{j}, t^{*}\right)\right) I_{k} z_{k}
$$


By definition, $t^{*}<t_{j}\left(z_{N}\right)$. Define $e_{N^{\rho}}^{\prime}=\left(R_{N^{\rho}}^{\prime}, a_{N^{\rho}}^{\prime}, g\right) \in \mathcal{E}$ to be a $\rho$-replica of $e_{N}$. We will derive a contradiction for $\rho$ large enough. Consider $e_{N^{\rho}}^{\prime \prime}=\left(R_{N^{\rho}}^{\prime}, a_{N^{\rho}}^{\prime \prime}, g\right) \in \mathcal{E}$, where $a_{N^{\rho}}^{\prime \prime}$ is such that $a_{k}^{\prime \prime}=\min a_{N^{\rho}}^{\prime}=a_{j}$ and $a_{i}^{\prime}=a_{i}^{\prime \prime}$ for all $i \neq k$. Define $z_{N^{\rho}}^{\rho^{\rho}}$ to be such that $z_{i}=z_{j}^{\rho}$, for all $i \in N$ and all $j \in \xi^{-1}(i)$. By RI, $z_{N^{\rho}}^{\rho} \in \varphi\left(e_{N^{\rho}}^{\prime}\right)$. By EFEA, we may write $\underline{t}\left(z_{N^{\rho}}^{\rho}\right), \underline{t}\left(z_{N^{\rho}}^{\prime \prime}\right)$ to denote the tax liability of the least talented in $e_{N^{\rho}}^{\prime}, e_{N^{\rho}}^{\prime \prime}$ respectively. Let $\lambda \equiv\left|N\left(\min a_{N^{\rho}}^{\prime}\right)\right|=\rho\left|N\left(\min a_{N}\right)\right|$. Letting $\tilde{N}=N^{\rho}-N\left(\min a_{N^{\rho}}\right)-\{k\}$, by PE, for all $z_{N^{\rho}}^{\prime \prime} \in \varphi\left(e_{N^{\rho}}^{\prime \prime}\right)$ we obtain

$$
t_{k}\left(z_{N^{\rho}}^{\rho}\right)+\lambda \underline{t}\left(z_{N^{\rho}}^{\rho}\right)+\sum_{i \in \tilde{N}} t_{i}\left(z_{N^{\rho}}^{\rho}\right)=(\lambda+1) \underline{t}\left(z_{N^{\rho}}^{\prime \prime}\right)+\sum_{i \in \tilde{N}} t_{i}\left(z_{N^{\rho}}^{\prime \prime}\right)=0 .
$$

Moreover, by AM, we have that

$$
t_{i}\left(z_{N^{\rho}}^{\rho}\right) \geq t_{i}\left(z_{N^{\rho}}^{\prime \prime}\right), \quad \text { for all } i \in \tilde{N} .
$$

From (3) and (4) we deduce that

$$
\underline{t}\left(z_{N^{\rho}}^{\rho}\right) \leq\left(1+\frac{1}{\lambda}\right) \underline{t}\left(z_{N^{\rho}}^{\prime \prime}\right)-\frac{1}{\lambda} t_{k}\left(z_{N^{\rho}}^{\rho}\right) .
$$

Moreover, by AM, we get

$$
\underline{t}\left(z_{N^{\rho}}^{\prime \prime}\right) \leq \underline{t}\left(z_{N^{\rho}}^{\rho}\right) \leq\left(1+\frac{1}{\lambda}\right) \underline{t}\left(z_{N^{\rho}}^{\prime \prime}\right)-\frac{1}{\lambda} t_{k}\left(z_{N^{\rho}}^{\rho}\right) .
$$

By letting $\rho$ tend to infinity we obtain $\underline{t}\left(z_{N^{\rho}}^{\prime \prime}\right) \simeq \underline{t}\left(z_{N^{\rho}}^{\rho}\right)>t^{*}$, which violates AM.

There are incompatibilities among the axioms above which are pertinent to our discussion. For some $g_{\circ} \in \mathcal{G}$, define $\left.\mathcal{E}\right|_{g=g_{\circ}}$ to be the sub-domain of $\mathcal{E}$ for which $g=g_{\circ}$.

Proposition 3. For any $g_{\circ} \in \mathcal{G}$, there exists no allocation rule $\varphi$, defined on $\left.\mathcal{E}\right|_{g=g_{\circ}}$, that satisfies Pareto Efficiency, Equal Welfare for Equal Preference and No-Envy among the Equally Able.

Fleurbaey and Maniquet[7] obtain the same incompatibility in the context of a production economy with exogenous marginal productivities. We corroborate their result, although one would have reasonably hoped otherwise. Interestingly enough, the solution of equalizing budget lines, although it violates Pareto Efficiency, has the property of satisfying both Equal Welfare for Equal Preference and Equal Outcome Frontier for Equal Ability. Apparently, by endogenizing productivities, because of Pareto Efficiency, we cannot improve on the conclusion of Fleurbaey and Maniquet[7]. As a consequence, equality of opportunity cannot be given a unique axiomatic definition. It needs to be treated as an elastic concept. Any compromise between the two principles of Compensation and Responsibility, whether it leans more towards the one or the other, will be treated as a legitimate stance on the matter.

Fleurbaey and Maniquet[8] prove that Pareto Efficiency and Ability Monotonicity are incompatible with Responsibility, in a similar model with exogenous productivities ${ }^{10}$. Proposition 4 is weaker, thus allowing for a potential compromise between weak collective ownership of ability, namely Ability Monotonicity, and Responsibility axioms.

Proposition 4. There exists no allocation rule $\varphi$ that satisfies Pareto Efficiency, No-Envy among the Equally Able and Ability Solidarity.

\footnotetext{
${ }^{10}$ Contrary to Fleurbaey and Maniquet[8], we assume a constant returns to scale production technology. In that respect we are less general than they are.
} 


\section{Allocation Rules}

In this section we propose allocation rules and discuss their implications. In the first part we discuss Responsibility-oriented allocation rules at the expense of Compensation. In the second part, we do the opposite.

4.1. Responsibility. The first allocation rule we will discuss is the laissez-faire allocation rule, illustrated in figure 3 .

Allocation Rule 1. An allocation rule $\varphi$ is called laissez-faire $\left(\varphi^{\text {lf }}\right)$ if and only if for all $e_{N} \in \mathcal{E}$, for all $z_{N} \in \varphi\left(e_{N}\right), z_{N}$ is Pareto Efficient and, moreover, for all $i, j \in N$ we have that $t_{i}\left(z_{N}\right)=t_{j}\left(z_{N}\right)=0$.

The axiomatization of laissez-faire allows us to evaluate its ethical viewpoint. Apart from complying fully with Responsibility, it demonstrates that excluding the possibility of redistribution across individuals with different innate abilities is compatible with Ability Monotonicity. Moreover, it satisfies Weak Consistency, a robustness axiom. If, for some economy, the bundle prescribed by the allocation rule is such that some individuals receive a zero transfer, the axiom requires that after removing these individuals, the bundles assigned to the rest remain unchanged. Thomson[21] reviews the implication of a series of axioms that relate to Weak Consistency.

Axiom 10. An allocation rule $\varphi$ satisfies Weak Consistency (WCON) if and only if for all $e_{N} \in \mathcal{E}$ and for all $z_{N} \in \varphi\left(e_{N}\right)$

$$
\exists M \subset N \text { s.t. } t_{i}\left(z_{N}\right)=0, \forall i \in M \Rightarrow z_{N}^{\prime} \in \varphi\left(R_{N \backslash M}, a_{N \backslash M}, g\right),
$$

where $z_{i}=z_{i}^{\prime}$ for all $i \in N-M$.

Weak Consistency plays an instrumental role in isolating laissez-faire within a more general class of rules that are founded on the same ethical premise.

Proposition 5. An allocation rule $\varphi$ satisfies Pareto Efficiency, Equal Outcome Frontier for Equal Ability, Ability Monotonicity and Weak Consistency if and only if it is the laissezfaire allocation rule $\varphi^{l f}$.

Proof. We omit the proof that $\varphi^{l f}$ satisfies the axioms. Take any $e_{N} \in \mathcal{E}$, and any $z_{N} \in \varphi\left(e_{N}\right)$. Let $M \in 2^{\mathbb{N}_{+}}$be such that $M \cap N=\emptyset$ and $|M|=|N|$. Let $e_{M \cup N}^{\prime}=$ $\left(R_{N \cup M}, s_{N \cup M}, g\right) \in \mathcal{E}$ be such that $a_{N \cup M}=\left(a_{N}, a_{N}\right)$ and $R_{N \cup M}=\left(R_{N}, R_{M}\right)$. Moreover, for all $i \in M, R_{i} \in R_{M}$ is represented by $u_{i}\left(c_{i}, l_{i}\right)=c_{i}-\theta l_{i}, \theta>0$ being sufficiently large. Hence, by PE, for all $z_{M \cup N}^{\prime} \in \varphi\left(e_{M \cup N}^{\prime}\right)$, for all $i \in M$ it must be $l_{i}=0$. By Proposition 1 we may invoke MSOA: any $z_{M \cup N}^{\prime} \in \varphi\left(e_{M \cup N}^{\prime}\right)$ must be such that, for all $i \in M, t_{i}\left(z_{M \cup N}^{\prime}\right) \geq 0$. Hence, by EFEA, for all $z_{M \cup N}^{\prime} \in \varphi\left(e_{M \cup N}^{\prime}\right)$, for all $i \in N$ we obtain $t_{i}\left(z_{M \cup N}^{\prime}\right) \geq 0$. By PE, these facts imply that for any $z_{M \cup N}^{\prime} \in \varphi\left(e_{N}^{\prime}\right)$ we have $t_{i}\left(z_{M \cup N}^{\prime}\right)=0$, for all $i \in N$. By WCON, there exists $z_{N}^{1} \in \varphi\left(e_{N}\right)$ such that $t_{i}\left(z_{N}^{1}\right)=0$, for all $i \in N$. By AM, if there exists $z_{N} \in \varphi\left(e_{N}\right)$ such that $z_{N} \neq z_{N}^{1}$, it must be that $z_{i} I_{i} z_{i}^{1}$, for all $i \in N$. Finally, by EFEA, for all $z_{N} \in \varphi\left(e_{N}\right)$ this latter fact implies that $t_{i}\left(z_{N}\right)=0$, for all $i \in N$.

Responsibility does not in general preclude redistribution. In fact, the main conclusion of this section will be to acknowledge this fact and, in addition, describe precisely the extent of redistribution that Responsibility will tolerate. Before we can do that, we need first to take some intermediate steps. 

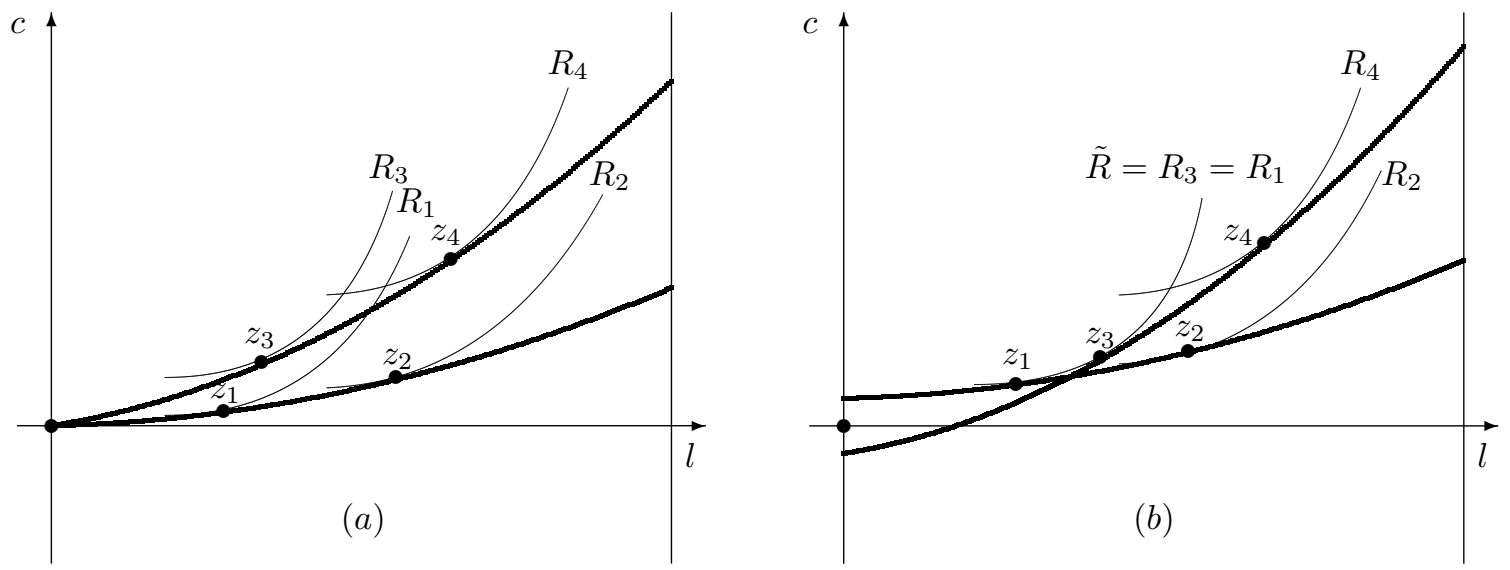

FiguRE 3. The two Responsibility-minded allocation rules: $\varphi^{l f}$ in part (a) and $\varphi^{\tilde{R}}$ in part (b). The economy comprises of 4 persons and, moreover, $R_{1}=R_{3}, R_{2}=R_{4}, a_{1}=a_{2}$ and $a_{3}=a_{4}$.

The axiom No Outcome Set Domination defends the idea that one's outcome frontier should not be be in the interior of the union of outcome sets of everyone else. Every individual should enjoy some consumption possibilities that are available only to him. The axiom forbids domination of outcome sets, namely a situation in which one outcome frontier lies everywhere below another (hence the name), for some economy and prescribed allocation. It is stronger than Equal Outcome Frontier for Equal Ability. Thomson[22] explores ideas similar in spirit. If one is to interpret consumption possibilities as opportunities, the axiom makes a lot of sense in terms of equality of opportunity. It should be stressed, however, that such an interpretation is biased towards Responsibility.

Axiom 11. An allocation rule $\varphi$ satisfies No Outcome Set Domination (NOSD) if and only if for all $e_{N} \in \mathcal{E}$ and all $z_{N} \in \varphi\left(e_{N}\right)$, there does not exist $i \in N$ such that

$$
O S\left(a_{i}, t_{i}\left(z_{N}\right)\right) \subset \bigcup_{j \in N-\{i\}} O S\left(a_{j}, t_{j}\left(z_{N}\right)\right) .
$$

It is instructive to note that No Outcome Set Domination bears an alternative statement : for all $e_{N} \in \mathcal{E}$ and all $z_{N} \in \varphi\left(e_{N}\right)$, there must exist $\tilde{R} \in \mathcal{R}$ such that for all $i, j \in N$

$$
m\left(\tilde{R}, O S\left(a_{i}, t_{i}\left(z_{N}\right)\right)\right) \tilde{I} m\left(\tilde{R}, O S\left(a_{j}, t_{j}\left(z_{N}\right)\right)\right) .
$$

Therefore, an alternative interpretation of the axiom is that it requires the equalization of welfare from the point of view of at least one preference relation in the admissible domain. Independently of the interpretation, No Outcome Set Domination is a strong requirement. However, Proposition 6 states that No Outcome Set Domination comes free if one adheres to Pareto Efficiency, Equal Outcome Frontier for Equal Ability and Ability Monotonicity.

Proposition 6. If an allocation rule $\varphi$ satisfies Pareto Efficiency, Equal Outcome Frontier for Equal Ability and Ability Monotonicity then it satisfies No Outcome Set Domination.

Proof. Suppose not, i.e. let there exist $e_{N} \in \mathcal{E}, z_{N} \in \varphi\left(e_{N}\right)$ and $j, k \in N$ such that the outcome frontier of individual $k$ at $z_{N}$ lies everywhere below that of individual $j$ at $z_{N}$. By EFEA, it is $a_{j} \neq a_{k}$. Suppose that $a_{k}>a_{j}$. This violates MSOA, which the axioms imply 
by Proposition 1. Suppose then that $a_{j}>a_{k}$. Let $e_{N}^{\prime}=\left(R_{N}, a_{N}^{\prime}, g\right) \in \mathcal{E}$, where $a_{j}^{\prime}=a_{k}$ and $a_{i}^{\prime}=a_{i}$ for all $i \in N-\{j\}$. Let $z_{N}^{\prime} \in \varphi\left(e_{N}^{\prime}\right)$. By AM, $t_{k}\left(z_{N}^{\prime}\right) \leq t_{k}\left(z_{N}\right)$. By EFEA, $t_{k}\left(z_{N}^{\prime}\right)=t_{j}\left(z_{N}^{\prime}\right)$. By assumption, $t_{j}\left(z_{N}\right)>t_{k}\left(z_{N}\right)$. Putting everything together we obtain $t_{j}\left(z_{N}^{\prime}\right)<t_{j}\left(z_{N}\right)$. By PE, $\sum_{i \in N} t_{i}\left(z_{N}\right)=\sum_{i \in N} t_{i}\left(z_{N}^{\prime}\right)$. Thus, $t_{j}\left(z_{N}^{\prime}\right)<t_{j}\left(z_{N}\right)$ implies that $\sum_{i \in N-\{j\}} t_{i}\left(z_{N}\right)<\sum_{i \in N-\{j\}} t_{i}\left(z_{N}^{\prime}\right)$. For this latter fact to be true there must exist some individual $i \in N$ for whom $t_{i}\left(z_{N}\right)<t_{i}\left(z_{N}^{\prime}\right)$, a violation of AM.

There is a relation $\succ$, to be read 'more averse to labour than', which underlies the set $\mathcal{R}$. It is transitive, yet not complete, as we have not made an assumption on the domain of preferences that will guarantee us a single crossing property in the leisure-consumption space.

Definition. For all $R, R^{\prime} \in \mathcal{R}$ we will write $R \succ R^{\prime}$ if and only if for all $a \in \mathbb{R}_{+}$, all $T \in \mathbb{R}$, all $(l, c) \in m(R, O S(a, T))$ and all $\left(l^{\prime}, c^{\prime}\right) \in m\left(R^{\prime}, O S(a, T)\right)$ we have $l^{\prime} \geq l$.

Given an economy $e_{N} \in \mathcal{E}$ we will generally be able to rank some individuals according to their aversion to labour. Let, for all $e_{N} \in \mathcal{E}, \mathcal{L}\left(e_{N}\right)=\left\{R \in R_{N}: \nexists R^{\prime} \in R_{N}\right.$ such that $R^{\prime} \succ$ $R\}$. This is the set of preferences that exhibit the highest degree of aversion to labour in the profile $R_{N}$. The set $\mathcal{L}\left(e_{N}\right)$ is non-empty and its cardinality will range from 1 to $|N|$, depending on the economy.

Allocation Rule 2. An allocation rule $\varphi$ belongs to the family of Reference Preference Egalitarian Equivalent rules ${ }^{11}\left(\varphi^{\tilde{R}}\right)$ if and only if for all $e_{N} \in \mathcal{E}$ there exists $\tilde{R} \in \mathcal{R}$ such that for all $z_{N} \in \varphi\left(e_{N}\right)$ and all $i, j \in N$

$$
m\left(\tilde{R}, O S\left(a_{i}, t_{i}\left(z_{N}\right)\right)\right) \tilde{I} m\left(\tilde{R}, O S\left(a_{j}, t_{j}\left(z_{N}\right)\right)\right) .
$$

Proposition 7. An allocation rule $\varphi$ satisfies Pareto Efficiency, Equal Outcome Frontier for Equal Ability, Ability Monotonicity and Replication Invariance if and only if $\varphi \in \varphi^{\tilde{R}}$ and, moreover, the reference parameter $\tilde{R}$ is such that for all $e_{N} \in \mathcal{E}$, either $\tilde{R} \in \mathcal{L}\left(e_{N}\right)$ or $\tilde{R} \succ R^{\prime}$ for all $R^{\prime} \in \mathcal{L}\left(e_{N}\right)$.

Proof. $\quad$ Step 1. If $\varphi$ satisfies the axioms then for all $e_{N}=\left(R_{N}, a_{N}, g\right) \in \mathcal{E}$, all $z_{N} \in \varphi\left(e_{N}\right)$ and all $k, j \in N$ such that $a_{k}=\min a_{N}$ and $a_{j}>\min a_{N}$ it must be

$$
m\left(R_{k}, O S\left(a_{j}, t_{j}\left(z_{N}\right)\right)\right) R_{k} z_{k} .
$$

Suppose not. Let there exist $e_{N} \in \mathcal{E}^{N}$ and $z_{N} \in \varphi(e)$ such that

$$
z_{k} P_{k} m\left(R_{k}, O S\left(a_{j}, t_{j}\left(z_{N}\right)\right)\right) \text {. }
$$

Define $t^{*}$ to be such that

$$
m\left(R_{k}, O S\left(a_{j}, t^{*}\right)\right) I_{k} z_{k}
$$

Let $e_{N^{\rho}}^{\prime}=\left(R_{N^{\rho}}, a_{N^{\rho}}^{\prime}, g\right) \in \mathcal{E}^{N^{\rho}}$ be a $\rho$-replica of $e_{N}$ and consider $e_{N^{\rho}}^{\prime \prime}=\left(R_{N^{\rho}}, a_{N^{\rho}}^{\prime \prime}, g\right) \in \mathcal{E}$, where $a_{N^{\rho}}^{\prime \prime}$ is such that $a_{k}^{\prime \prime}=a_{j}$ and $a_{N^{\rho}-\{k\}}^{\prime}=a_{N^{\rho}-\{k\}}^{\prime \prime}$. Define $z_{N^{\rho}}^{\rho}$ to be such that $z_{i}=z_{j}^{\rho}$, for all $i \in N$ and all $j \in \xi^{-1}(i)$. By RI, $z_{N^{\rho}}^{\rho} \in \varphi\left(e_{N^{\rho}}^{\prime}\right)$. By EFEA, we may write $t^{a_{j}}\left(z_{N^{\rho}}^{\rho}\right), t^{a_{j}}\left(z_{N^{\rho}}^{\prime \prime}\right)$ to denote the tax liability of all the individuals with innate ability equal to $a_{j}$ in $e^{\prime}$ and $e^{\prime \prime}$ respectively. Let $\lambda \equiv\left|N\left(\min a_{N^{\rho}}\right)\right|=\rho\left|N\left(\min a_{N}\right)\right|$. Let $\tilde{N}=$ $N^{\rho}-N\left(\min a_{N^{\rho}} ; e^{\prime}\right)-\{k\}$. By PE, for all $z_{N^{\rho}}^{\prime \prime} \in \varphi\left(e_{N^{\rho}}^{\prime \prime}\right)$ we obtain

$$
t_{k}\left(z_{N^{\rho}}^{\rho}\right)+\lambda t^{a_{j}}\left(z_{N^{\rho}}^{\rho}\right)+\sum_{i \in \tilde{N}} t_{i}\left(z_{N^{\rho}}^{\rho}\right)=(\lambda+1) t^{a_{j}}\left(z_{N^{\rho}}^{\prime \prime}\right)+\sum_{i \in \tilde{N}} t_{i}\left(z_{N^{\rho}}^{\prime \prime}\right),
$$

\footnotetext{
${ }^{11}$ Refer to figure 3 , part (b) for an illustration.
} 
Moreover, by AM, it must be

$$
\sum_{i \in \tilde{N}} t_{i}\left(z_{N^{\rho}}^{\rho}\right) \leq \sum_{i \in \tilde{N}} t_{i}\left(z_{N^{\rho}}^{\prime \prime}\right)
$$

By (5),(6) we deduce that

$$
t^{a_{j}}\left(z_{N^{\rho}}^{\rho}\right) \geq\left(1+\frac{1}{\lambda}\right) t^{a_{j}}\left(z_{N^{\rho}}^{\prime \prime}\right)-\frac{1}{\lambda} t_{k}\left(z_{N^{\rho}}^{\rho}\right) .
$$

Moreover, by AM, we may write

$$
t^{a_{j}}\left(z_{N^{\rho}}^{\prime \prime}\right) \geq t^{a_{j}}\left(z_{N^{\rho}}^{\rho}\right) \geq\left(1+\frac{1}{\lambda}\right) t^{a_{j}}\left(z_{N^{\rho}}^{\prime \prime}\right)-\frac{1}{\lambda} t_{k}\left(z_{N^{\rho}}^{\rho}\right) .
$$

By letting $\rho$ tend to infinity we obtain $t^{a_{j}}\left(z_{N^{\rho}}^{\prime \prime}\right) \simeq t^{a_{j}}\left(z_{N^{\rho}}^{\rho}\right)<t^{*}$, which violates AM.

Step 2. Let $A$ be the set of values of $a$ such that $\bigcup_{a \in A} N(a)=N$. In the following argument we will assume that $|A| \geq 2$. The proof is trivial for $|A|=1$. Let $\varphi$ satisfy the axioms. By Proposition 6, we may invoke NOSD. Thus, for all $e_{N} \in \mathcal{E}$, all $z_{N} \in \varphi\left(e_{N}\right)$ there exists $\tilde{R} \in \mathcal{R}$ such that

$$
m\left(\tilde{R}, O S\left(a_{i}, t_{i}\left(z_{N}\right)\right)\right) \tilde{I} m\left(\tilde{R}, O S\left(a_{j}, t_{j}\left(z_{N}\right)\right)\right),
$$

for any $i, j \in N$. Consider any $e_{N}=\left(R_{N}, a_{N}, g\right) \in \mathcal{E}$ and any $z_{N} \in \varphi\left(e_{N}\right)$ and suppose that $R^{l} \succ \tilde{R}$, for some $R^{l} \in R_{N}$. By Proposition 2 we may appeal to LSOA in order to conclude that for any $i \in N$ with $R_{i}=R^{l}$ it must be $a_{i}=\min a_{N}$. Given the convexity of $\tilde{R}$, this implies a violation of the condition formulated in Step 1.

The reference parameter can be interpreted as a measure of the redistribution which the rule effects (redistribution here being possible only among individuals with different innate abilities). Consider some economy $e_{N} \in \mathcal{E}$ and any two reference preferences $\tilde{R}^{\prime}, \tilde{R}^{\prime \prime} \in \mathcal{R}$ such that $\tilde{R}^{\prime \prime} \succ \tilde{R}^{\prime} \succ R$, for all $R \in \mathcal{L}\left(e_{N}\right)$. For any $z_{N}^{\prime} \in \varphi^{\tilde{R}^{\prime}}\left(e_{N}\right)$, any $z_{N}^{\prime \prime} \in \varphi^{\tilde{R}^{\prime \prime}}\left(e_{N}\right)$ it will be $\sum_{i \in N} \max \left\{0, t_{i}\left(z_{N}^{\prime}\right)\right\} \geq \sum_{i \in N} \max \left\{0, t_{i}\left(z_{N}^{\prime \prime}\right)\right\}$. This is illustrated in figure 4 , where we depict a simple two-person economy. The reference parameters $\tilde{R}^{\prime}, \tilde{R}^{\prime \prime}$ are chosen to be linear in order to facilitate the exposition. The two individuals have the same preferences which, in addition, exhibit no aversion to labour. Finally, $a_{2}>a_{1}$. One can see that $\varphi^{\tilde{R}^{\prime}}$ effects more redistribution than $\varphi^{\tilde{R^{\prime \prime}}}$.

Another noteworthy point is that the reference parameter can be chosen in a way that makes the rule accommodate some very mild form of Compensation. If everyone in the economy had the same preferences, welfare would ideally be equalized.

Axiom 12. An allocation rule $\varphi$ satisfies Equal Welfare for Uniform Preference if and only if for all $e_{N} \in \mathcal{E}$ and all $z_{N} \in \varphi\left(e_{N}\right)$

$$
R_{i}=R_{j} \text {, for all } i, j \in N \Rightarrow z_{i} I_{i} z_{j} \text {, for all } i, j \in N \text {. }
$$

In the example of figure 4 the economy is such that $R_{1}=R_{2}=\mathcal{L}\left(e_{N}\right)$. In any such economy, in order to satisfy Equal Welfare for Uniform Preference, we need to have $\tilde{R}=\mathcal{L}\left(e_{N}\right)$.

There are two conclusions to be drawn. First, some $\tilde{R} \in \mathcal{L}\left(e_{N}\right)$ is a natural candidate for the reference parameter. It effects the most redistribution the economy will allow for. Second, the economy may not allow for much redistribution at all. If all preferences in $\mathcal{L}\left(e_{N}\right)$ are very averse to labour, we cannot escape laissez-faire.

We have thus concluded with the view of equality of opportunity that leans towards responsibility. We now turn to the other extreme and place full weight on compensation. 


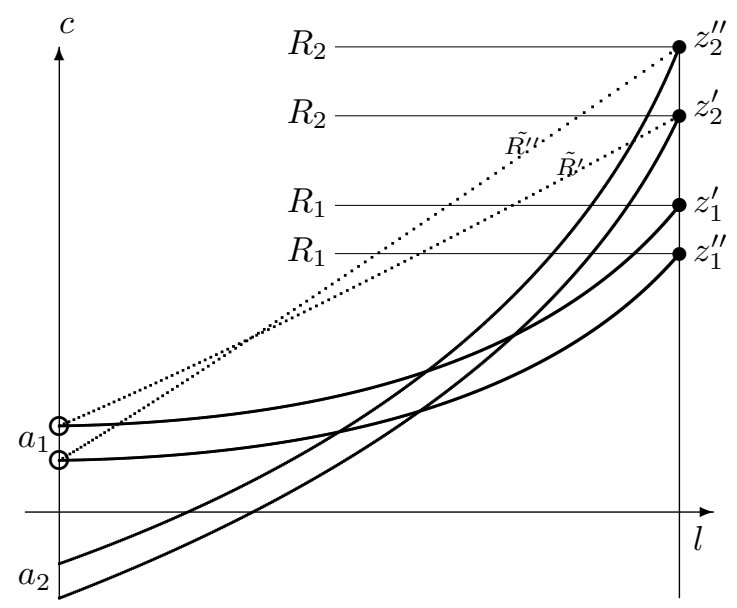

FiguRE 4. Redistribution decreases as the 'laziness' of the reference parameter increases.

4.2. Compensation. The first allocation rule we propose is in the tradition of Pazner and Schmeidler [13]. It selects allocations that would be envy-free if everyone in the society had the same innate ability (see figure 5(a) for an illustration).

Allocation Rule 3. An allocation rule $\varphi$ is an $\tilde{a}$-egalitarian equivalent rule $\left(\varphi^{\tilde{a}}\right)$ if and only if for all $e_{N} \in \mathcal{E}$, all $z_{N} \in \varphi\left(e_{N}\right)$ and all $i \in N$ there exists $\tilde{a} \in \mathbb{R}_{+}$such that

$$
\left.z_{i} I_{i} m\left(R_{i}, O S(\tilde{a}, 0)\right)\right) \text {. }
$$

Existence follows from Assumption 1, clause (b).

Proposition 8. An allocation rule $\varphi$ satisfies Pareto Efficiency, Ability Solidarity and Minimal Self-Ownership of Ability if and only if it is the $\tilde{a}$-egalitarian equivalent rule $\varphi^{\tilde{a}}$.

Proof. Let an allocation rule $\varphi$ satisfy the axioms. Suppose that for some $e_{N}=$ $\left(R_{N}, a_{N}, g\right) \in \mathcal{E}$, some $z_{N} \in \varphi\left(e_{N}\right)$, there exists $\tilde{\tilde{a}}, \tilde{a} \in \mathbb{R}_{+}$, with $\tilde{\tilde{a}}<\tilde{a}$, and $i, j \in N$ such that

$$
z_{i} I_{i} m\left(R_{i},(O S(\tilde{a}, 0))\right)
$$

and

$$
\left.z_{j} I_{j} m\left(R_{j}, O S(\tilde{\tilde{a}}, 0)\right)\right) .
$$

Consider $e_{N}^{\prime}=\left(R_{N},(\bar{a}, \ldots, \bar{a}), g\right) \in \mathcal{E}$, with $\tilde{a}>\bar{a}>\tilde{\tilde{a}}$. Consider an allocation $z_{N}^{\prime}$ such that for all $k \in N$

$$
z_{k}^{\prime}=m\left(R_{k}, O S(\bar{a}, 0)\right) .
$$

By PE and MSOA, $z_{N}^{\prime} \in \varphi\left(e_{N}^{\prime}\right)$. By the construction of $z_{N}^{\prime}$, we have $z_{i} P_{i} z_{i}^{\prime}$ and $z_{j}^{\prime} P_{j} z_{j}$. Hence, AS is violated.

A factor to take into account is that Ability Solidarity is compatible with Minimal SelfOwnership of Ability even when our priority is Compensation. If one is to favor Limited SelfOwnership of Ability, one should have to give up on Ability Monotonicity. This possibility is presented below (See figure 5(b) for an illustration). 
Allocation Rule 4. An allocation rule $\varphi$ is an $\min a_{N}$-equivalent rule ( $\varphi^{\underline{a}}$ ) if and only if for all $e_{N} \in \mathcal{E}$, all $z_{N} \in \varphi\left(e_{N}\right)$, all $i \in N$ and all $j \in N\left(\min a_{N}\right)$

$$
z_{i} I_{i} m\left(R_{i}, O S\left(a_{j}, t_{j}\left(z_{N}\right)\right)\right) .
$$

In particular, in abandoning solidarity-type axioms while still insisting on full compensation, we may satisfy further libertarian objectives. Policy is often focused on the most disadvantaged individuals. Pareto Efficiency and Limited Self-Ownership of Ability are natural limits to the resources that can be directed to the least able.

Proposition 9. An allocation rule $\varphi$, that belongs to the class of allocation rules satisfying Pareto Efficiency and Limited Self-Ownership of Ability, has the property of maximizing, for all $e_{N} \in \mathcal{E}$, the welfare of the least able, if and only if $\varphi=\varphi \underline{\text { a }}$.

Proof. Suppose, first, that $\varphi$ satisfies the axioms and $\varphi \neq \varphi^{\underline{a}}$. This implies that there exists $e_{N} \in \mathcal{E}, z \in \varphi\left(e_{N}\right), k \in N$ and $j \in N\left(\min a_{N}\right)$ such that

$$
z_{k} P_{k} m\left(R_{k}, O S\left(\min a_{N}, t_{j}\left(z_{N}\right)\right)\right) \text {. }
$$

Moreover, by LSOA, we have that for all $i \in N, t_{i}\left(z_{N}\right)=t_{j}\left(z_{N}\right)$. Hence, $k \in N-$ $N\left(\min a_{N}\right)$. There exists a Pareto Efficient allocation $z_{N}^{\epsilon}$ compatible with the vector of lump-sum transfers $t\left(z_{N}^{\epsilon}\right)$, where $t_{k}\left(z_{N}^{\epsilon}\right)=t_{k}\left(z_{N}\right)-\epsilon$ and $t_{i}\left(z_{N}^{\epsilon}\right)=t_{i}\left(z_{N}\right)+\frac{\epsilon}{|N|-1}$, for all $i \in N$. For $\epsilon$ positive and small enough we have that $z_{N}^{\epsilon}$ respects LSOA. In addition, for all $i \in N\left(\min a_{N}\right)$, we obtain $z_{i}^{\epsilon} P_{i} z_{i}$. Therefore, $\varphi$ does not maximize the welfare of the least able in the economy $e_{N}$.

Conversely, suppose that there exists $e_{N} \in \mathcal{E}, z \in \varphi^{\underline{a}}\left(e_{N}\right), z_{N}^{\prime} \in \varphi\left(e_{N}\right)$ and $k \in N\left(\min a_{N}\right)$ such that $z_{k}^{\prime} P_{k} z_{k}$. By PE, this implies that $t_{k}\left(z_{N}^{\prime}\right)>t_{k}\left(z_{N}\right)$. By LSOA, $t_{k}\left(z_{N}^{\prime}\right)=t_{i}\left(z_{N}^{\prime}\right)$ and $t_{k}\left(z_{N}\right)=t_{i}\left(z_{N}\right)$ for all $i \in N$. By PE, there exists $j \in N \backslash N\left(\min a_{N}\right)$ such that $t_{j}\left(z_{N}^{\prime}\right)<t_{j}\left(z_{N}\right)$. By definition

$$
z_{j} I_{j} m\left(R_{j}, O S\left(\min a_{N}, t_{k}\left(z_{N}\right)\right)\right) .
$$

Hence,

$$
m\left(R_{j}, O S\left(\min a_{N}, t_{k}\left(z_{N}^{\prime}\right)\right)\right) P_{j} z_{j}^{\prime},
$$

which constitutes a violation of LSOA. Therefore, the welfare of the least able in $z_{N}$ is the maximum the axioms will allow for.

No allocation rule we reviewed in this section precludes allocations that transfer resources from the untalented to the talented. We demonstrate this in two examples depicted in figure 5. The a -egalitarian equivalent rule is depicted first. The reference parameter is denoted $\tilde{a}$. The portrayed economy consists of three individuals. One may notice that although $a_{2}>a_{1}$ the allocation rule prescribes $t_{1}\left(z_{1}, z_{2}, z_{3}\right)=0<t_{2}\left(z_{1}, z_{2}, z_{3}\right)$. The following figure deals with the min $a_{N}$-equivalent rule. Once more, although $a_{3}^{\prime}>a_{2}^{\prime}>a_{1}^{\prime}$ we obtain $t_{3}\left(z_{1}^{\prime}, z_{2}^{\prime}, z_{3}^{\prime}\right)>0$ while $t_{2}\left(z_{1}^{\prime}, z_{2}^{\prime}, z_{3}^{\prime}\right)<0$.

Another noteworthy feature of these allocation rules is the way in which they treat hardworking individuals. Any generic allocation they propose induces a partition of the set of agents into contributors and receivers. Of two receivers with the same talent, the one who is more prone to labour will end up receiving more. As investment in human capital ceteris paribus decreases with the laziness of one's preference, under Assumption 1, investment in human capital is rewarded. Surprisingly, among the contributors and within the same talent level the effect is reversed. If one is a highly talented individual and thus a contributor, one will envy an individual with the same innate ability if and only if he is more averse to 

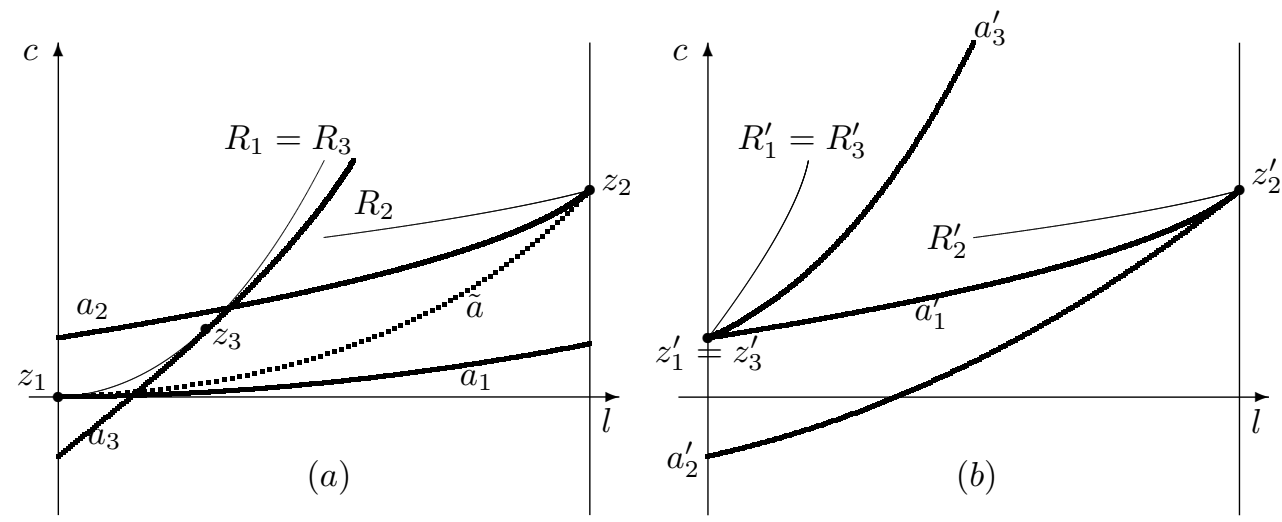

Figure 5. The two Compensation-minded allocation rules: $\varphi^{\tilde{a}}$ in part (a) and $\varphi^{\underline{a}}$ in part (b). Both rules allow for a transfer from a less talented to a more talented individual to occur.

labour. The view of equality of opportunity that is based on Compensation rewards laziness among contributors and punishes it among receivers.

\section{Concluding Remarks}

In our analysis we assumed full information. This served to expose the implications of Pareto Efficiency. It leaves a distinct mark on all our results. Investment in human capital needs be determined by the profile of preferences and innate abilities. This is what Pareto Efficiency dictates. A policy, for instance, that would aim at guaranteeing all individuals a minimum positive amount of investment in human capital would clash with efficiency. To see this, consider an individual who is infinitely averse to labour. Any amount spent in building earning capabilities for that particular individual is an amount wasted. The broader conclusion is that differences in innate abilities will translate into differences in marginal productivities. The concept of Equality of Opportunity we put forward acknowledges this premise.

The allocation rules we characterized rationalize policy, in the form of transfers and, in particular, associate it with ethical principles. The Responsibility-minded allocation rule $\varphi^{\tilde{R}}$ we presented sheds light on both the scope and the extent of redistribution. The amount of resources transfered in the direction of the less talented is profile-dependent. Still, the $\varphi^{\tilde{R}}$ would appear to justify public intervention as opposed to laissez-faire. On the Compensation side, the rules we propose strive to make a subset of the consumption space equally accessible to all. We provide two solutions that accomplish this and discriminate between them on a moral basis. We summarize our results in the table below. 


\begin{tabular}{lccccc}
\hline & $\varphi^{\tilde{a}}$ & $\varphi^{\underline{a}}$ & $\varphi^{l f}$ & $\varphi^{\tilde{R}}$ \\
& & & & \\
\hline Pareto Efficiency & + & + & + & + \\
EWEP & + & + & - & - \\
EFEA & - & - & + & + \\
NEEA & - & - & + & + \\
Ability Solidarity & + & - & - & - \\
Ability Monotonicity & + & - & + & + \\
Min. Self-Own/ship of Ability & + & + & + & + \\
Lim. Self-Own/ship of Ability & - & + & + & + \\
W. Consistency & - & - & + & - \\
\hline
\end{tabular}

The question that yet confronts us is how these results carry through to the second best analysis. Let us imagine that innate abilities and investment in human capital are observable, whereas preferences are not. There exists a promising strand of literature that has generalized allocation rules into social welfare orderings. Unlike an allocation rule that selects the best social outcomes in a certain domain, a social welfare ordering ranks all the elements of the domain. Therefore, orderings can be maximized under incentive compatibility constraints (Fleurbaey and Maniquet[9] discuss income taxation from this perspective in a Mirlees model with preference heterogeneity and exogenous skills). The whole exercise is interesting for the additional reason that investment profiles, if assumed to be observable, become a parameter of important informative value. Needless to say, the second best analysis will provide richer policy recommendations.

\section{Appendix}

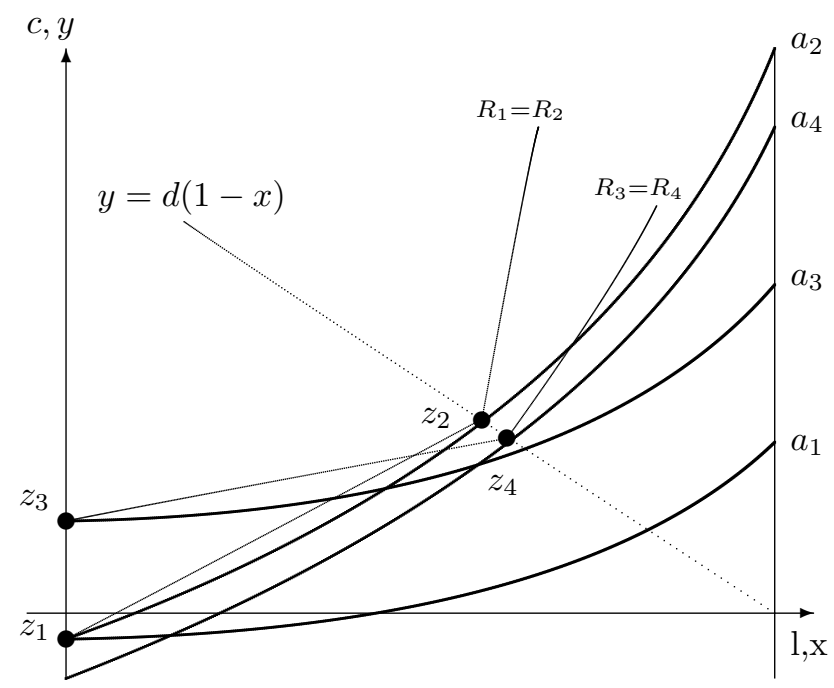

Figure 6. Pareto Efficiency, Equal welfare for Equal Preference and No Envy among the Equally Able are incompatible.

Proof of Proposition 3. We present a counter-example. Fix $g_{\circ} \in \mathcal{G}$ and suppose that $\varphi$, defined on $\left.\mathcal{E}\right|_{g=g_{\circ}}$, satisfies the axioms. Consider $e_{N}=\left.\left(R_{N}, a_{N}, g_{\circ}\right) \in \mathcal{E}\right|_{g=g_{\circ}}$, where 
$N=\{1,2,3,4\}, a_{1}=a_{3}<a_{2}=a_{4}$ and preferences are homothetic. In addition, for some $\alpha>\gamma \geq 0, \beta>\delta \geq 0$ and $d>0$, the utility representation of preferences is given by

$$
u_{i}\left(c_{i}, l_{i}\right)=c_{i}-\left\{\begin{array}{ccc}
\alpha l_{i} & \text { if } & c_{i} \leq d\left(1-l_{i}\right) \\
\beta l_{i} & \text { if } \quad & c_{i}>d\left(1-l_{i}\right)
\end{array}\right.
$$

for $i=1,2$, while

$$
u_{i}\left(c_{i}, l_{i}\right)=c_{i}-\left\{\begin{array}{lll}
\gamma l_{i} & \text { if } & c_{i} \leq d\left(1-l_{i}\right) \\
\delta l_{i} & \text { if } \quad & c_{i}>d\left(1-l_{i}\right)
\end{array}\right.
$$

for $i=3,4$. Given the fact that the outcome frontier is increasing and convex, for any $g \in \mathcal{G}$, a menu of parameters $(d, \alpha, \beta, \gamma, \delta)$ exists such that, by PE, for all $\left.e_{N} \in \mathcal{E}\right|_{g=g_{\circ}}$ and all $z_{N} \in \varphi\left(e_{N}\right), l_{i}=0$ for $i=1,3$ and $l_{i}=\frac{c_{i}-d}{d}$ for $i=2,4$. Suppose that $R_{N}$ is constructed so as to accomplish this effect. For all $z_{N} \in \varphi\left(e_{N}\right)$, by EWEP, we need to have $z_{1} I_{i} z_{2}$ for $i \in\{1,2\}$ and $z_{3} I_{i} z_{4}$ for $i \in\{3,4\}$. Figure 6 depicts such an allocation. This generates a case of 'double envy' among individuals with the same ability. Refer to figure 6: $z_{2} P_{4} z_{4}$ and $z_{3} P_{1} z_{1}$. Hence, by EWEP and PE, for all $z_{N} \in \varphi\left(e_{N}\right)$ it can be either $t_{1}\left(z_{N}\right)=t_{3}\left(z_{N}\right)$ and $t_{2}\left(z_{N}\right)>t_{4}\left(z_{N}\right)$ or $t_{2}\left(z_{N}\right)=t_{4}\left(z_{N}\right)$ and $t_{1}\left(z_{N}\right)<t_{3}\left(z_{N}\right)$. Thus, NEEA is contradicted.

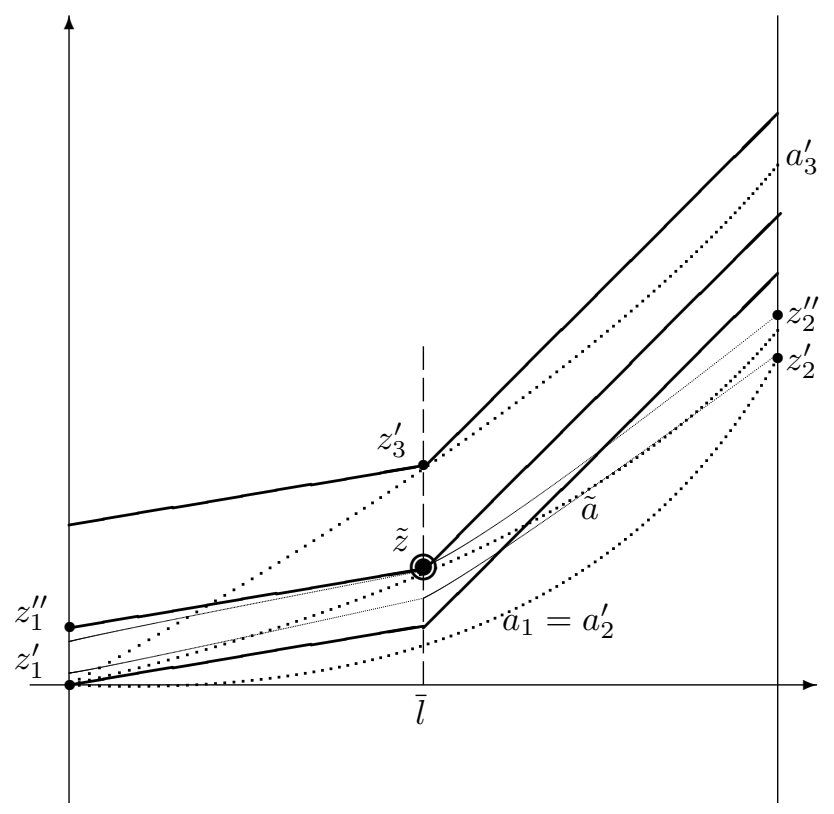

Figure 7. Pareto Efficiency, No-Envy among the Equally Able and Ability Solidarity are incompatible.

Proof of Proposition 4. We present a counter-example. Suppose that an allocation rule $\varphi$ satisfies the axioms. Let $N=\{1,2,3\}$ and consider $e_{N}=\left(R_{N},(\tilde{a}, \tilde{a}, \tilde{a}), g\right) \in \mathcal{E}$ such that $R_{i}$ is quasi-linear and, in addition, for some $0<\widehat{\alpha}<\widehat{\gamma}<\widehat{\delta}<\widehat{\beta}$, the utility representation of $R_{i}$ is given by:

for $i=1,3$, while

$$
u_{i}\left(c_{i}, l_{i}\right)=c_{i}-\left\{\begin{array}{ccc}
\widehat{\alpha} l_{i} & \text { if } & l_{i} \leq \bar{l} \\
\widehat{\beta} l_{i} & \text { if } & l_{i}>\bar{l}
\end{array}\right.
$$

$$
u_{i}\left(c_{i}, l_{i}\right)=c_{i}-\left\{\begin{array}{lll}
\widehat{\gamma} l_{i} & \text { if } & l_{i} \leq \bar{l} \\
\widehat{\delta} l_{i} & \text { if } & l_{i}>\bar{l}
\end{array}\right.
$$

for $i=2$. Refer to figure 7. Thick lines represent $R_{1}=R_{3}$, while thin $R_{2}$. The choice of parameters is such that for all $z_{N} \in \varphi\left(e_{N}\right)$, by PE, $l_{i}=\bar{l}$ for all $i \in N$. Therefore, 
by $\operatorname{NEEA}(\tilde{z}, \tilde{z}, \tilde{z})=\varphi\left(e_{N}\right)$. Let $e_{N}^{\prime}=\left(R_{N}, a_{N}^{\prime}, g\right) \in \mathcal{E}$, with $a_{1}^{\prime}=a_{2}^{\prime}<\tilde{a}$ and $a_{3}^{\prime}>\tilde{a}$. Figure 7 illustrates the outcome frontiers with dotted curves. The function $g$ is such that the curvature of the outcome fortier decreases in $a$. Hence, PE dictates that for all $z_{N}^{\prime} \in$ $\varphi\left(e_{N}^{\prime}\right), l_{1}=0, l_{2}=1$ and $l_{3}=\bar{l}$. The allocation $z_{N}^{\prime}=\left(z_{1}^{\prime}, z_{2}^{\prime}, z_{3}^{\prime}\right)$, depicted in Figure 7 , is Pareto Efficient in $e_{N}^{\prime}$, with $t_{i}\left(z_{N}^{\prime}\right)=0$, for all $i \in N$. Moreover, by construction, $\left\|z_{1}^{\prime}-z_{1}^{\prime \prime}\right\|+\left\|z_{2}^{\prime}-z_{2}^{\prime \prime}\right\|=\left\|\tilde{z}-z_{3}^{\prime}\right\|$. By AS and the fact that PE requires balanced transfers, for any $z_{N} \in \varphi\left(e_{N}^{\prime}\right)$ it must be $t_{1}\left(z_{N}\right)=\left\|z_{1}^{\prime}-z_{1}^{\prime \prime}\right\|, t_{2}\left(z_{N}\right)=\left\|z_{2}^{\prime}-z_{2}^{\prime \prime}\right\|$ and $t_{3}\left(z_{N}\right)=\left\|\tilde{z}-z_{3}^{\prime}\right\|$. Therefore $\left(z_{1}^{\prime \prime}, z_{2}^{\prime \prime}, \tilde{z_{N}}\right) \in \varphi\left(e_{N}^{\prime}\right)$. This violates NEEA: $z_{1}^{\prime \prime} P_{2} z_{2}^{\prime \prime}$.

\section{REFERENCES}

[1] Angrist J., Krueger A.B. 1991, "Does Compulsory School Attendance Affect Schooling and Earnings?', Quarterly Journal of Economics 106: 979-1014.

[2] Arneson, R. 1989, "Equality and Equal Opportunity for Welfare", Philosophical Studies, 56: 77-93.

[3] Becker, G., Tomes, N. 1986, "Human capital and the Rise and Fall of Families", Journal of Labour Economics, 4: S1-S47.

[4] Dworkin, R. 1981, "What is Equality? Part 1: Equality of Welfare", Philosophy and Public Affairs, 10: $185-246$.

[5] Dworkin, R. 1981, "What is Equality? Part 1: Equality of Resources", Philosophy and Public Affairs, 10: $283-345$.

[6] Fleurbaey M. 1995, "Three solutions to the compensation problem", Journal of Economic Theory 65: 505-521.

[7] Fleurbaey M., Maniquet F. 1996, "Fair Allocation with Unequal Production Skills : The No Envy Approach to Compensation", skills" Mathematical Social Sciences 32: 71-93.

[8] Fleurbaey M., Maniquet F. 1999, "Cooperative Production with Unequal Skills : The Solodarity Approach to Compensation", Social Choice and welfare, 16: 569-583

[9] Fleurbaey M., Maniquet, F. 2007, "Help the Low Skilled or Let the Hard Working Thrive: A Study of Fairness in Optimal Income Taxation", Journal of Public Economic Theory, 9(3): 467-500.

[10] Fleurbaey M., Maniquet, F. forthcoming, "Compensation and Responsibility", K.J. Arrow, A.K. Sen and K. Suzumura(eds) Handbook of Social Choice.

[11] Hanushek E.A. 1971, "The Economics of Schooling : Production and Efficiency in Public Schools", Journal of Economic Literature 24: 1141-1177.

[12] Loury G. 1981, "Intergenerational Transfers and the Distribution of Earnings", Econometrica, 49: 843-867.

[13] Pazner A. And Schmeidler D. 1974, "Egalitarian Equivalent Allocations: A New Concept of Economic Equity" Quarterly Journal of Economics, 92: 671-687.

[14] Maniquet F., Sprumont Y. 2005, "Welfare Egalitarianism in Non-Rival Environments", Journal of Economic Theory 120: 155-174.

[15] Mirlees J. 1971, "An Exploration in the Theory of Optimal Income Taxation", Review of Economic Studies 38: 175-208.

[16] Moulin H. 1990, "Joint Ownership of a Convex Technology", Review of Economic Studies 57: 439-452.

[17] Moulin H. 1990, "Uniform Externalities: Two Axioms for Fair Allocation", Journal of Public Economics 43: 305-326.

[18] Moulin H., Roemer J.E. 1989, "Public Ownership of the External World and Private Ownership of Self", Journal of Political Economy 97: 347-367.

[19] Nozick R. 1974, "Anarchy, State and Utopia", Basic Books, New York.

[20] Thomson W. 1983, "Equity in exchange Economies", Journal of Economic Theory 29: 217-244.

[21] Thomson W. 1988, "A Study of Choice Correspondences in Economies with a Variable Number of Agents", Journal of Economic Theory 46: 237-254.

[22] Thomson W. 1994, "Notions of Equal or Equivalent Opportunities", Social Choice and Welfare 11: $137-156$.

[23] Rawls J. 1971, "A Theory of Justice", Harvard University Press, Cambridge.

[24] Roemer JE 1986, "Equality of Resources Implies equality of Welfare", Quarterly Journal of Economics 101: $751-784$

[25] Roemer JE 1998, "Equality of Opportunity", Harvard University Press. 